\title{
Les dérapages des relations publiques
}

\author{
Bernard Dagenais, professeur titulaire au \\ Département d'information et de communication, \\ Université Laval \\ Bernard.Dagenais@com.ulaval.ca
}

\begin{abstract}
Un professionnel des relations publiques peut-il échapper aux pressions de ses patrons lorsqu'ils décident de taire la vérité, de construire des scénarios autour de demi-vérités, de pratiquer la désinformation? La réponse est non. Dès lors, le professionnel des relations publiques devient un mercenaire au service de gens sans scrupules, ou se transforme en avocat et défend la cause de ses employeurs sans souci du bien public. Comment empêcher que cette situation perdure? Trop souvent le professionnel des relations publiques doit se soumettre ou se démettre. Pour éviter le dérapage continuel de la profession des relations publiques, il faut absolument que les associations professionnelles acceptent de livrer un combat sur la place publique contre les dérives du métier. Or, rien n'indique qu'elles soient prêtes à livrer ce combat.
\end{abstract}


Au début des années 2010, la télévision de Radio-Canada présentait une télé série télévisée intitulée Mirador, mettant en vedette un cabinet de relations publiques dirigé par des professionnels sans scrupules. Dans le contexte de la diffusion de cette série, Radio-Canada a réalisé une émission avec des professionnels chevronnés des relations publiques pour essayer de comprendre ce qu'était vraiment ce métier et si la série reflétait une part de vérité. Les professionnels invités à cette émission se sont campés dans deux positions bien distinctes. Certains ont affirmé que la profession de relationniste s'appuie sur un code d'éthique qui condamne totalement les pratiques de Mirador; et d'autres voient davantage la profession de relationniste comme celle de l'avocat qui doit par tous les moyens légaux sauver la réputation de son client.

$\mathrm{Au}$ même moment, paraissait l'essai de Michel Dumas (2010) dans lequel il exposait de façon très fouillée les raisons qui militent en faveur de la professionnalisation du métier de relationniste, pour éviter les trop nombreux dérapages que connaît la profession, provenant entre autres des doreurs d'images (les spin doctors).

Nous voulons exposer dans cet article le fossé qui existe entre la notion d'éthique que prônent les professionnels des relations publiques et la dérive d'une certaine pratique des relations publiques où le professionnel se transforme en mercenaire pour qui tous les moyens sont bons, sous le regard silencieux des associations représentant la profession.

\section{La recherche d'un consensus}

Quelle est la première responsabilité d'un professionnel des relations publiques? C'est de faire connaître, aimer et adopter les biens, services et idées de l'entreprise qui l'engage. Au-delà de ce premier niveau tactique, il doit s'engager dans des voies stratégiques et savoir gérer l'image, les enjeux, les risques et les crises qui marquent la vie de l'entreprise. Pour réussir sur ces deux tableaux, il doit nécessairement démontrer de l'empathie et de l'écoute envers ses divers publics et ceux-ci doivent exprimer une complicité certaine avec l'entreprise.

Selon Grunig (1992), le modèle idéal de cette situation s'articulerait autour de la communication symétrique bidirectionnelle. Ce qui signifie que l'entreprise parle à son public, l'écoute et s'ajuste à sa réaction. Nous sommes alors dans un univers d'ouverture, de complicité et d'écoute attentive.

Dans la réalité, les seules occasions où cette communication peut exister, c'est lorsque l'émetteur et le récepteur partagent les mêmes valeurs et discutent ensemble de la façon de les exploiter. Ou plus souvent lorsque le citoyen/consommateur n'a ni les connaissances, ni le recul nécessaire pour juger de la véracité des informations qui lui sont communiquées. La plupart du temps, l'émetteur et le récepteur ne sont pas sur la même longueur d'onde, sont opposés idéologiquement ou stratégiquement, ou sont de mauvaise foi. L'entreprise est donc en mode de guerre et 
non de séduction, en mode d'attaque et non d'écoute, en mode de suspicion et non de complicité. Et c'est aussi vrai à l'externe qu'à interne.

À l'externe, croire qu'une entreprise, dont le bilan en matière d'environnement ou de pollution souffre de multiples carences, puisse entretenir des relations harmonieuses avec les groupes environnementalistes, relève de l'utopie. Les deux groupes s'affrontent parce qu'ils ne partagent pas les mêmes valeurs, les mêmes intérêts et les mêmes préoccupations. Ils sont en guerre pour gagner la bataille de l'opinion publique et le support des autorités politiques. Et les règles de la guerre diffèrent des règles de l'amitié.

À l'interne, le fossé existant entre les attentes patronales et les demandes syndicales peut être si infranchissable qu'on ira jusqu'à la grève, au lock-out prolongé et même à la fermeture de l'entreprise à défaut de s'entendre.

Il existe certes à l'externe comme à l'interne des situations où peut s'exercer une communication bidirectionnelle symétrique, parce qu'il existe une réelle harmonie entre les interlocuteurs, mais nous tenons pour acquis qu'il s'agit de circonstances d'exception. Les luttes de pouvoir que se livrent les individus tant à l'interne qu'à l'externe des organisations, les positions irréconciliables, parfois appuyées par des comportements irresponsables de l'une ou l'autre des parties rendent impossible une entente harmonieuse. Nous sommes sur le terrain de la guerre: guerre de concurrence, guerre des conditions de travail, guerre pour la protection de l'environnement, guerre politique, guerre des prix, guerre des religions, guerre d'intimidation ou de harcèlement, nous sommes toujours en milieu hostile. L'objectif des protagonistes n'est pas d'implanter une communication complice, mais de gagner la bataille sinon la guerre sur le terrain de l'opinion publique. Et en s'appuyant sur cette opinion favorable, il leur faut en même temps essayer d'infléchir les décisions des autorités par l'intervention de lobbyistes (Buchholtz 1989) et par certaines générosités douteuses. On peut donc se retrouver devant des entreprises qui affirment sur la place publique se préoccuper de la protection de l'environnement et favoriser le développement durable; et en même temps se battre férocement pour empêcher toute législation ou réglementation d'imposer quelques contraintes en matière de diminution de la pollution, par exemple.

Si l'on reconnaît que les relations entre les partenaires sur la place publique relèvent davantage de l'art de la guerre que de celui de la collaboration, la dernière chose à faire est de partager avec l'ennemi ses plans de combat. Le contrôle de l'information et la censure s'impose. Pour contrer les effets de la désinformation et de la diabolisation de l'autre, il ne s'agit pas de présenter l'autre joue, mais de se défendre sur le même terrain. Dans toute guerre, chaque protagoniste affirme qu'il livre une guerre juste et que l'autre est un usurpateur et il tente de le démontrer (Dagenais 2008).

Désormais, sur la place publique, avec les malversations et les scandales de la grande entreprise, la corruption des pouvoirs publics, le refus des politiciens 
d'accepter les critiques, la présence des groupes d'intérêts et l'émergence de groupes de citoyens responsables, on ne peut plus parler de possibilité d'échanges harmonieux entre les parties, tellement les positions sont opposées.

La moindre information, même factuelle, est à la merci de conflits de valeurs. Par exemple, l'annonce d'un spectacle culturel, donnant les dates et le lieu des représentations et la liste des artistes en présence, se répète plusieurs fois par semaine sans aucun problème. Mais la présentation du Cycle des femmes de Sophocle par Wajdi Mouawad, avec la présence de Bertrand Cantat, d'abord à Avignon en 2010 et au Théâtre du Nouveau Monde au printemps 2011, au Québec, a créé un tel tollé que Cantat s'est désisté. Il ne s'agit pas là d'une communication bidirectionnelle symétrique. Il s'agit là d'une guerre d'idées ou l'une a gagné et l'autre a cédé.

Cette guerre d'idées se joue avec des stratégies de relations publiques, de publicité, de lobbying, de propagande, de désinformation, d'intimidation. Les plus riches jouissent d'un pouvoir de conviction sonnant et trébuchant, les plus pauvres bénéficient d'une large sympathie auprès d'un certain public et de certains journalistes, comme l'ont démontré le groupe des indignés partout à travers le monde en 2012.

Force nous est de constater que la théorie de Grunig repose sur une vision idéale de la pratique des relations publiques au $21^{\mathrm{e}}$ siècle. Le code de déontologie de la Société québécoise des professionnels en relations publiques présente le même type d'ambiguïté. Il est l'expression d'une vision angélique de la réalité. Le premier article précise que «[les] membres doivent exercer leur profession conformément à l'intérêt public ». Or les professionnels des relations publiques œuvrent tous pour des entreprises et des organisations qui ont des intérêts bien particuliers. Même les professionnels qui travaillent pour un gouvernement ou un organisme public doivent se souvenir qu'ils œuvrent d'abord et avant tout pour un parti politique élu.

\section{L'avocat de l'image de l'entreprise devant l'opinion publique}

Le professionnel des relations publiques est un expert dont l'un des mandats est de tout faire pour aider son entreprise à conquérir les espaces publics qu'elle convoite, pour l'aider à se sortir des impasses dans lesquelles elle est plongée et pour protéger son image contre toute attaque et contre ses propres erreurs de parcours.

De ce fait, le professionnel des RP agit en quelque sorte comme un avocat auprès de son client. Son but premier est de sortir gagnant des enjeux qu'il doit gérer. Il représente son entreprise devant le tribunal de l'opinion publique, celle-ci étant alimentée par des adversaires coriaces et des idéalistes convaincus. Et il doit affronter les journalistes qui exigent qu'elle se confesse chaque jour de tous ses petits et grands péchés, sinon elle sera accusée de manque de transparence ou de 
lèse-journalisme, celui-ci étant défini comme le refus d'un acteur de se plier aux exigences des journalistes. Le professionnel des relations publiques doit défendre à tout prix l'image de l'entreprise, celle-ci représentant son capital le plus important.

Avocat ou professionnel des relations publiques, ce sont deux professions qui visent les mêmes buts avec des approches différentes. Dès lors, pourquoi existe-t-il dans le milieu des relations publiques, une école de pensée qui refuse absolument toute identification avec le métier d'avocat (Versailles 2011)?

Le professionnel des relations publiques, comme l'avocat, vend ses services pour gérer les enjeux auxquels fait face un client ou un employeur et l'aide à occuper l'espace public de la manière la plus rentable pour lui. Les deux métiers invoquent dans leur code d'éthique qu'il faut que cela se fasse dans la recherche du bien public et du respect de la dignité humaine. Dans les deux cas, le client est parfois en mode de combat parce qu'il veut conquérir un nouvel espace, parfois en mode de défense parce qu'il subit des attaques de l'extérieur.

Qu'est-ce qui agace le professionnel des relations publiques dans sa comparaison avec le métier d'avocat et pourquoi une telle comparaison fait-elle parfois grincer des dents?

C'est que l'image du métier d'avocat ne correspond pas à celle que les professionnels des relations publiques s'attribuent. Un avocat doit tout faire pour empêcher son client, parfois coupable, d'être condamné au risque de faire condamner un innocent. Son rôle n'est pas la recherche de la vérité, mais de défendre son client en utilisant tous les artifices de l'argumentation pour présenter de façon crédible sa version des faits et pour rendre douteuse la version de l'autre. Il doit créer de la confusion.

Il faut se rappeler l'histoire de l'Afro-Américain Rodney King, tabassé par quatre policiers blancs en 1991. La scène fut filmée et diffusée sur toutes les chaînes de télévision américaines. Or les avocats des quatre policiers blancs ont présenté l'altercation filmée comme la preuve que King avait agressé les policiers et que ceux-ci s'étaient défendus. Les policiers "agressés » ont été acquittés par un jury blanc, ce qui provoqua des émeutes importantes à Los Angeles. Rappelons-nous aussi le cas d'OJ Simpsons, accusé d'avoir assassiné son ex-épouse et le compagnon de celle-ci en 1994. Il était acquitté l'année suivante à la suite d'un long procès très controversé et très médiatisé. Les avocats qui ont défendu les quatre policiers et OJ Simpsons sont considérés comme des professionnels du droit de haut niveau. Dans les deux cas, il n'était pas question de rechercher la vérité, mais bien de gagner l'enjeu du procès. Ils ont réussi à créer assez de confusion dans l'esprit du jury pour faire acquitter leurs clients qui seront par ailleurs condamnés dans un procès subséquent.

La firme de relations publiques qui a défendu l'intégrité de l'ex-premier ministre canadien Brian Mulroney soupçonné d'avoir reçu un pot de vin dans l'affaire Airbus a gagné la bataille de l'opinion publique et M. Mulroney a reçu des compensations 
du gouvernement du Canada qui l'avait soupçonné à tort de ces malversations. Mais quelques années plus tard, M. Mulroney déclarait avoir reçu en argent comptant quelque $200000 \$$ de l'homme d'affaires Schreiber, celui-là même qui était impliqué dans l'affaire Airbus. La firme de relations publiques avait pourtant réussi aux yeux de la justice et de l'opinion publique à faire croire à l'innocence de M. Mulroney.

L'avocat comme le professionnel des relations publiques reçoit le mandat de protéger l'intégrité de son client. Il n'est jamais à la recherche de la vérité mais du seul bien-être de son client. C'est cette vision trop crue de la réalité qui choque certains relationnistes.

\section{Les dérives du métier}

Dans tous les secteurs de l'activité humaine, les relations publiques exercent un rôle ambigu.

\subsection{L'information en politique}

La Fédération professionnelle des journalistes du Québec publiait en novembre 2004 et en novembre 2005 deux dossiers noirs sur la communication gouvernementale, dans lesquels elle dénonçait toutes les difficultés que rencontraient les journalistes dans l'exercice de leur travail. Ces dossiers noirs reviennent de façon sporadique depuis quelque 30 ans.

Que reprochent les journalistes à la communication des gouvernements? Mensonges, omissions, opacité, retenues indues de l'information, utilisation abusive de la loi d'accès à l'information pour retarder la diffusion d'information ou pour l'extirper de son véritable contenu, directives paralysantes pour les communicateurs de l'administration publique, en somme la communication est au service de la personne politique et non au service du public.

Sur le plan municipal, en novembre 2010, la Fédération professionnelle des journalistes du Québec rendait public un dossier intitulé: Dossier noir de l'information municipale dans lequel elle dénonçait les conditions de travail de ses membres dans les municipalités : limite dans l'accès à l'information, intimidation, entraves, menaces et mesures de rétorsion économique étaient désignés comme constituant une situation d'hostilité du monde politique municipal face aux médias.

Que font les professionnels des relations publiques qui travaillent comme attachés de presse, attachés politiques, directeurs ou agents d'information, directeurs ou chargés de compte des firmes de relations publiques reliés au monde politique? Ils participent à l'opacité de l'information, à la désinformation et au mensonge institutionnalisé. Qu'ils le souhaitent ou non, ils sont au service d'une machine à trahir la vérité, ils en sont les complices. 
En politique, la culture du mensonge s'impose. L'ex-ministre Claude Morin (1984, p. 305), dans un texte intitulé «La décision politique », expliquait la difficulté pour une personnalité politique de prendre en compte les intérêts des citoyens dans le processus décisionnel. D'abord, pour Morin, la décision, c'est le pouvoir. Dans un système démocratique ouvert, les acteurs jouent un rôle prédominant. Le gouvernement, le parti d'opposition, les groupes de pression cherchent à influencer le processus décisionnel. «Dans la décision se trouve une constante implicite: les choix, les orientations, les programmes conçus par les ministres, les députés du parti au pouvoir n'obéissent souvent à aucune rationalité véritable; tout y est, la plupart du temps, arbitraire et subjectif. Le but est le maintien au pouvoir de ceux qui s'y trouvent déjà ou son acquisition par ceux qui y aspirent».

Le même discours, venant de Claude Ryan, ancien directeur du prestigieux quotidien Le Devoir à Montréal et devenu ministre, apporte du crédit aux réflexions de Morin. Il est impossible à un homme politique de dire toute la vérité. C'est le ministre de l'Éducation Claude Ryan qui le disait en avril 1987. «Les hommes politiques ne peuvent pas dire toute la vérité et ceux qui prétendent le faire sont des imposteurs », a-t-il déclaré. Le ministre expliquait qu'il était difficile dans ses contacts avec la presse de cacher la vérité sans mentir. Il a reconnu que «c'est parfois difficile d'y arriver tant les questions persistantes des journalistes sont parfois tellement près du cœur des réalités. Ça prend tout notre art, dit-il, pour rester dans le vrai sans le dire au complet. On est incapable au pouvoir [de ne pas jouer avec la vérité], ça ne sert à rien de se faire des théories » (Ryan 1997).

Claude Morin affirmait sans ambages en présentant son livre Lendemains piégés à l'automne 1988 que les politiciens sont tous des menteurs.

Dans son livre Pour en finir avec octobre (1982, p. 52), Francis Simard livre ces propos sur la façon de s'exprimer des hommes politiques. À la suite de la conférence de presse qu'a tenue le premier ministre du Québec, Robert Bourassa, au lendemain de l'enlèvement de Laporte, lors des événements d'octobre 1970 au Québec, où le ministre Laporte a finalement été tué, l'auteur raconte qu'ils étaient restés sous l'impression que le gouvernement avait l'intention de négocier. Une personne même avait été nommée pour agir comme négociateur au nom du gouvernement.

Nous avions peut-être mal écouté ce qu'avait vraiment dit Robert Bourassa. Par après, en relisant le texte de son discours, je me suis aperçu qu'au fond il n'avait rien accepté... Peut-être aussi que le langage de Robert Bourassa, le supposé langage politique, ce n'est pas le nôtre. Nous n'y sommes pas habitués. Une façon de parler, d'enligner (sic) des mots qui laissent sous-entendre bien des choses mais qui, en réalité, ne disent rien. Tu laisses entendre que tu es prêt à accepter de négocier, mais les mots sont choisis de telle façon que ça peut vouloir dire l'inverse aussi, le contraire. Façon diplomatique de gagner du temps... de nous leurrer. 
Il n'a pas été le seul à ne pas comprendre. Les commentateurs de Radio Canada avaient interprété de leur côté que le gouvernement refusait de négocier, le soir même, du discours de Bourassa. Et ils l'ont dit. Puis ils se sont fait reprocher d'avoir mal interprété les paroles de Bourassa. On comprit les jours suivants qu'ils les avaient bien interprétées.

L'éditorialiste du quotidien La Presse, André Pratte, dénonçait en mai 2009, la malhonnêteté intellectuelle des libéraux provinciaux, alors au pouvoir au Québec

Une vidéo présentée aux militants... retraçait l'histoire de l'hydroélectricité au Québec. Le PLQ [Parti libéral du Québec] a réussi le tour de force d'y raconter la nationalisation de l'électricité sans dire un mot de René Lévesque. Ministre libéral des Ressources naturelles au début des années 60, M. Lévesque a mené pendant des mois une campagne publique en faveur de la nationalisation. C'est cette campagne qui a convaincu les Québécois, puis le premier ministre Lesage de prendre cet audacieux pari. On a beau être partisan, il y a des limites à la malhonnêteté intellectuelle.

M. René Lévesque fonda ensuite le Parti Québécois qui occupa le pouvoir au Québec de 1975 à 1985.

Aux trois paliers de gouvernement, il y a des centaines de professionnels des relations publiques qui se plient à ce jeu de la désinformation, soit par appétit du pouvoir et ils se comportent comme des avocats pour qui tous les moyens sont bons pour réussir, soit par obligation professionnelle pour ne pas perdre leur emploi, et ils se comportent comme de dociles mercenaires.

Les professionnels et les firmes de RP au service du politique participent ainsi à un dérapage certain de la profession. L'affaire du faux témoignage des couveuses au Koweït en est un bien triste exemple. Rappelons les faits tirés de Wikipédia (Affaires des couveuses au Koweit, s.d.).

«Le 10 octobre 1990, lors de l'invasion du Koweït par les forces armées irakiennes de Saddam Hussein, [...] une jeune femme koweïtienne témoigne, les larmes aux yeux devant une commission du Congrès des États-Unis. » «Monsieur le président, messieurs les membres de ce comité, je m'appelle Nayirah et je reviens du Koweït. Ma mère et moi étions au Koweït le 2 août pour passer de paisibles vacances. Ma sœur aînée avait accouché le 29 juillet et nous voulions passer quelque temps au Koweït auprès d'elle. [...] Pendant que j'étais là, j'ai vu les soldats irakiens entrer dans l'hôpital avec leurs armes. Ils ont tiré sur les bébés des couveuses, ils ont pris les couveuses et ont laissé mourir les bébés sur le sol froid. J'étais horrifiée. Je ne pouvais rien faire et je pensais à mon neveu qui était né prématuré et aurait pu mourir ce jour-là lui aussi. [...] Les Irakiens ont tout détruit au Koweït. Ils ont vidé les supermarchés de nourriture, les pharmacies de médicaments, les usines de matériel médical, ils ont cambriolé les maisons et torturé des voisins et des amis. J'ai vu un de mes amis après qu'il a été 
torturé par les Irakiens. Il a 22 ans, mais on aurait dit un vieillard. Les Irakiens lui avaient plongé la tête dans un bassin, jusqu'à ce qu'il soit presque noyé. Ils lui ont arraché les ongles. Ils lui ont fait subir des chocs électriques sur les parties sensibles de son corps. Il a beaucoup de chance d'avoir survécu. »

« Ce témoignage a beaucoup ému l'opinion publique internationale et a contribué à ce qu'elle soutienne l'action des puissances occidentales contre les armées de Saddam Hussein lors de la guerre du Golfe. En fait, ce témoignage était entièrement faux. La jeune fille était la fille de l'ambassadeur du Koweït à Washington. L'association Citizens for a Free Kuwait, mise sur pied par le gouvernement du Koweït exilé avait commandé cette campagne à la compagnie de relations publiques Hill \& Knowlton ».

Une personnalité politique peut-elle survivre sans un spin doctor (Dagenais 2006) ? Nous avons évoqué plus haut que le politicien admet qu'il doit mentir. Il va donc s'entourer de conseillers en communication, les spin doctors, que l'on traduit en français par doreurs d'image, qui vont faire tourner (to spin) les faits, les images, les événements de façon à favoriser les desseins de son initiateur. C'est une forme de propagande où les mensonges, les demi-vérités et la désinformation constituent les éléments de base. L'invention des armes de destruction massive chez Saddam Hussein relève du spin doctor.

Dumas (2010) dénonce à plusieurs reprises ces professionnels de la communication sans aucune morale ni aucun sens éthique qui nourrissent l'image négative qui colle au dos du professionnel des relations publiques.

\subsection{L’information dans le monde économique}

Tout n'est pas mensonge dans le domaine économique. Mais on rencontre le mensonge partout. Les actes du colloque «Contredire l'entreprise » (Catellani, Libaert \& Pierlot 2010) en apportent de multiples exemples. Le Média Center for Democracy démontre dans son bulletin hebdomadaire comment la grande entreprise utilise les approches les plus discutables pour se construire une image tout à fait contraire à la réalité. Le greenwashing (Notebaert et Séjeau, 2010) et l'astrosurfing (Austin, 1996) sont autant de techniques de mensonge abondamment utilisées par les conseillers en communication de ces entreprises. À cela s'ajoute la pratique de la responsabilité sociale des entreprises où se confondent vertu et fausse représentation. À ce sujet, l'industrie crée des concours pour récompenser des entreprises pour leur engagement social. Par exemple, au Québec, Korn/Ferry International, la firme de relations publiques National et le Magazine L'Actualité, organise le concours «Prix québécois de l'entreprise citoyenne » visant à reconnaître des gestes accomplis par des entreprises dont le siège social est au Québec et qui contribuaient à l'éthique des affaires et au développement durable. Mais en même temps, en France, le groupe écologiste «Les amis de la Terre» décerne le prix 
Pinocchio du développement durable pour dénoncer le décalage qu'il peut y avoir entre les discours publics et les comportements réels des entreprises françaises en matière de développement durable.

Enfin, la création de groupes de façade par la grande entreprise est destinée à sa face même à tromper le public. Il s'agit d'une stratégie de communication qui consiste à créer de toutes pièces un groupe qui porte un nom qui laisse croire que le groupe veut défendre une cause donnée, alors que le groupe est financé par des entreprises qui visent exactement le contraire (Beder 1998; Stauber et Rampton 2004; Pfau et al 2007; Bero et al 2007; Palenchar et al 2009; Agoravox 2011).

\subsection{Le rôle des relations publiques}

Greenswashing, astrosurfing, responsabilité sociale, groupe de façade, toutes ces activités sont créées et soutenues par des firmes et des professionnels de relations publiques. La première responsabilité d'un professionnel en RP est de satisfaire son patron/client et de chercher la complicité de son public cible. Ce n'est donc pas d'honorer un code d'éthique que trop de monde transgresse, ni de rechercher la transparence et encore moins de rechercher la communication bidirectionnelle symétrique. De toute façon, Libaert (2003) a bien démontré que la transparence pour une entreprise était suicidaire.

Dès lors, où se situent les professionnels des relations publiques? Des avocats dévoués, des mercenaires sans scrupule, des experts en techniques de communication, des apôtres au service d'un mieux-être collectif et à la défense d'un code d'éthique? Ils sont tout cela en même temps. Ce qui faisait dire à Dagenais $(1999$, p. 211) que le professionnel des relations publiques était un schizophrène.

\section{Le silence de la profession}

Que dit la profession sur les dérives commises par les professionnels des relations publiques?

- À la Société québécoise des professionnels en relations publiques (SQPRP), il n'existe aucun comité désigné pour étudier les plaintes relatives au manquement au code d'éthique et au cours des dernières décennies, aucun blâme n'a été prodigué ni à des membres en règle de la SQPRP, ni à des professionnels des RP qui auraient dérogé aux principes d'éthique de la profession.

- Comme la majorité des professionnels de RP ne sont pas membres de la SQPRP, celle-ci ne se sent pas mandatée pour gérer sur la place publique les comportements fautifs. Donc, personne ne gère les dérapages des professionnels en relations publiques, membres ou non membres de la SQPRP.

- Par ailleurs, trop d'écrits sur les relations publiques restent discrets sur le fossé existant entre l'adhésion de principe au code d'éthique de la SQPRP et les 
pratiques douteuses poursuivies par les professionnels des RP. Dumas (2010), par exemple, fait une nette distinction entre les relationnistes honnêtes et les doreurs d'image (spin doctors), oubliant que chaque relationniste est un Janus portant en même temps les deux faces du métier.

- Ce métier s'exerce dans un contexte de grande compétitivité et à une époque où trop de partenaires ont perdu tout sens moral et civique. Les théories sur lesquelles s'appuie le métier, comme celle de Grunig, relèvent d'un certain angélisme et masquent la réalité de l'exercice de la profession. C'est en vertu par ailleurs de cette théorie que toute comparaison avec le métier d'avocat semble inacceptable (Versailles 2011). Mais l'approche de Grunig est-elle une théorie ou une doctrine? Pour Edgar Morin (1991, p. 133), une théorie est un système ouvert qui évolue au gré des nouvelles connaissances. Une doctrine est un système fermé qui s'impose envers et contre toutes démonstrations. La façon dont certains relationnistes posent en évangile la thèse de Grunig nous laisse croire qu'on en a fait une doctrine infaillible.

\section{Conclusion}

Dans ce contexte, qui donc va stopper les dérives de la profession? Aucun professionnel des relations publiques ne peut, à lui seul, freiner dans son milieu de travail, le manque de scrupule de trop nombreux dirigeants politiques, économiques et socioreligieux qui ont recours à ses services.

Il reste donc aux associations professionnelles de se tenir debout et de dénoncer ces dérives, de les condamner sur la place publique et de soutenir les efforts des professionnels qui individuellement tentent de résister à la pression énorme des acteurs sans conscience.

Or, il faut bien le reconnaître, les associations professionnelles ferment les yeux sur ces dérapages et se contentent d'affirmer qu'il existe un code d'éthique interdisant toute malversation. Mais jamais sur la place publique furent critiquées par les associations professionnelles les dérives de certaines agences de relations publiques et de nombreux professionnels des relations publiques.

Accepter que les professionnels des relations publiques soient des mercenaires ou des avocats - et que dès lors, ils représentent sur la place publique les entreprises qui les paient - n'est pas indigne de la profession. Les professionnels des relations publiques ne sont détenteurs d'aucun contenu, contrairement à des professions comme les avocats (le droit), les ingénieurs (le génie), les médecins (la médecine), l'historien (l'histoire). En relations publiques, comme en publicité, en marketing et en journalisme, le professionnel est un spécialiste d'emballage dans lequel il présente sur la place publique un contenu qui ne lui appartient pas, qu'il doit apprendre à connaître ou qu'il doit créer et qu'il doit vendre. 
Le professionnel des RP est alors extrêmement satisfait lorsqu'il réussit l'impossible tâche de redonner un nouveau souffle de vie à l'entreprise ou à l'individu qui lui a confié son destin fragile ou son développement en péril. Ce sont là les véritables défis de la profession. Mercenaire sans loi, avocat sans foi, ou apôtre avec joie, le professionnel des relations publiques vend sa force de travail et son intelligence à celui qui le paie. Le seul qui résiste à l'appât du gain est celui qui est investi d'une morale intériorisée et non d'un code d'éthique corporatif.

Dès lors, le cynisme qui entoure la pratique de cette profession est presque encouragé par les associations professionnelles qui n’osent pas intervenir sur la place publique pour dénoncer les abus dont nous avons fait état, car trop de leurs membres sont à ce sujet en porte à faux.

\section{Bibliographie}

Affaire des couveuses (s.d.). Dans Wikipedia. Répéré le 14 janvier 2012 à http://fr.wikipedia.org/wiki/Affaire des couveuses au Koweit

Agoravox, Les Front Groups, des outils de désinformation de l'industrie, (11.06.2011). Repéré le 14 janvier 2012 à http://www.agoravox.fr/actualites/economie/article/les-front-groups-des-outilsde-95704

Apollonio, D., and Bero, L. (2007). The Creation of Industry Front Groups: The Tobacco Industry and "Get Government Off Our Back". American Journal of Public Health, (97), 419-427.

Austin, A. (1996). How the West is Won: Astroturf lobbying and the wise use Movement. The American Prospect, (25) (mars-avril).

Beder, Sharon (1998), Public Relations' Role in Manufacturing Artificial Grass Roots Coalitions, Public Relations Quarterly, 43 (2), 20-23.

Bero, A. Lisa, Apollonio, Dorie, (March 2007). The Creation of Industry Front Groups: The Tobacco Industry and "Get Government Off Our Back".American Journal of Public Health, 93(3).

Buchholz, Rogene (1989). Business Environment and Public Policy. Implications for management and strategy formulation. Englewood Cliffs (N.J.): Prentice Hall.

Catellani, A. et Pierlot, J-M., (dir) (2010). Contredire l'entreprise. Louvain, Belgique, Presses universitaires de Louvain.

Dagenais, B. (1999).Le métier de relationniste. Québec, Québec, Les Presses de l'Université Laval. 
Dagenais, B. (2006, décembre). Une personnalité politique peut-elle survivre sans un spin doctor?.Revue française de Marketing, cahier 210, 27 - 38.

Dagenais, B. (2008).L'Éloge de la violence, France : Éditions de l'Aube.

Dumas, M. (2010).Les relations publiques, une profession en devenir. Québec, Québec. Les Presses de l'université du Québec.

Grunig, J.E. (1992), Excellence in Public Relations and Communication Management, Hillsdale : N.J. Lawrence Erlbaum Associates.

Libaert, T. (2003), La Transparence en trompe-l'œil. Paris, France: Éditions Descartes et Cie.

Morin, C. (1984). La décision politique. Dans A. Riverin (dir), Le management des affaires publique, Boucherville, Québec : Gaétan Morin.

Morin, C. (1988).Lendemains piégés, Montréal : Éditions du Boréal.

Morin, E. (1991).La Méthode 4. Les idées, leur habitat, leur vie, leurs mours, leur organisation, Paris : Éditions du Seuil.

Notebaert, J-F., et Wilfrid, S. (2010), Écoblanchiment. Quand les $4 X 4$ sauvent la planète, Paris : Les petits matins.

Palenchar, M. J. et Fitzpatrik, K. R. (2009), Secret Persuaders : Ethical and Rhetorical Perspectives on the Use of Public Relations Front Groups. Dans Heath, R. L., Toth E. L., \& Waymer D., Ed.) Rhetorical and Critical Approaches to Public Relations II, (272-289). New York : Routledge.

Pfau, M., M. Haigh, M., Sims, J., Wigley, S. (2007).The Influence of Corporate Front-Group Stealth Campaigns, Communication Research, 34 (73).

Pratte, A. (2009), Les grands imposteurs, La Presse, 26 mai. Répéré à http://www.lapresse.ca/debats/editoriaux/andre-pratte/200905/26/01-859810les-grands-imposteurs.php, le 2 mars 2013.

Ryan, C. (1987), Le journal de Québec, 28 avril.

Simard, F. (1982), Pour en finir avec octobre. Montréal, Québec, Éditions Alain Stanké

Stauber, J., Rampton Sheldon (2004), L'industrie du mensonge, lobbying, communication, publicités et médias. Marseille, France. Éditions Agone.

Versailles, G. (2011).Vérité, mensonge et professionnalisme en relations publiques, série de cinq articles publiés dans REGARDS RP, le bulletin électronique de la Société québécoise des professionnels en relations publiques, les 26 et 30 mai, et les 10,15 et 22 juin. 